\title{
Optical characterization of a polymer micro-optical light coupler for silicon channels
}

\author{
Heidi Ottevaere, ${ }^{* 1}$ Sara Van Overmeire, ${ }^{1}$ Jorge Albero, ${ }^{2}$ Lukasz Nieradko, ${ }^{2}$ Christophe Gorecki ${ }^{2}$ and Hugo \\ Thienpont ${ }^{1}$ \\ ${ }^{l}$ Vrije Universiteit Brussel, Dept. of Applied Physics and Photonics (TONA), Brussels Photonics Team (B-PHOT), \\ Pleinlaan 2, B-1050 Brussel, Belgium \\ ${ }^{2}$ FEMTO-ST (UMR CNRS 6174), Département MN2S, UFR ST, 16 Route de Gray, 25030 Besançon Cedex, France
}

\begin{abstract}
We present a micro-optical system for UV/VIS absorbance detection in silicon microfluidic channels, which consists of a micro-optical light coupler placed on top of the silicon fluidic channel to probe the molecules under test with laser light. We demonstrate the proof-of-concept of this microfluidic light probe by measuring standard samples of coumarin 102 dye with concentrations between $0.6 \mu \mathrm{M}$ and $6 \mathrm{mM}$. Calibrating the system yields a detection limit of $4.3 \mu \mathrm{M}$. To conclude, we show that the concept of this microfluidic detection system is generic in that it can be applied at different positions on different microfluidic channel configurations.
\end{abstract}

In the past decades the development of practical microfluidic systems for biochemical analysis has evolved rapidly. However their applications have been limited to laboratory prototypes without widespread routine use in clinical or high-throughput applications [1]. This is partly due to technological limitations in two parts of the sample analysis cycle: sample preparation and detection. In particular, since the beginning, detection has been one of the main challenges in microfluidics. Indeed, very sensitive techniques must be employed to detect the limited amount of molecules available in the nanoliter detection volumes. Optical detection is one of the techniques capable of providing sufficient sensitivity but still it is commonly accomplished by bulky and expensive microscopes located off-chip. Miniaturized and integrated optical detection systems are therefore needed to fully exploit the potential advantages of microfluidic devices. The key detection technique in conventional biochemical techniques of analysis is the UV/VIS absorbance detection. Absorbance detection requires the propagation of an ultraviolet (UV) or visible (VIS) light beam through a type of microfluidic channel where the amount of absorbed light can be quantified. Since silicon absorbs UV and VIS light, this is often done by using optical fibers or waveguides to bring light to the channel and to collect nonabsorbed light [2].

In this letter we present a different approach to absorbance measurements in silicon microfluidic channels; namely an out-of-plane technique where the probing light enters and exits the sample perpendicularly to the plane of the chip. Such out-of-plane configurations have already been proposed for absorbance detection [3-
5], but in this work we have also miniaturized the optics needed to couple light in and out of the detection cell and we have integrated fibers to connect the optics to the source and the detector. As such a compact micro-optical detection unit is created. The system was also designed in such a way that fluorescence detection could be added to the microfluidic chip as additional functionality. The schematic of the system is shown in Fig. 1.

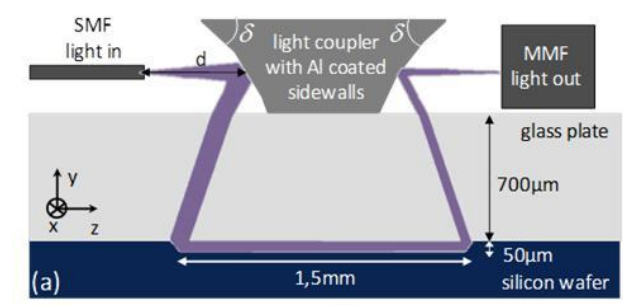

Fig. 1. Schematic of a single silicon channel detection unit with a polymer light coupler.

We place a polymer micro-optical light coupler on which a metallic coating is deposited on top of the silicon wafer to couple light in and out of the fluidic channel. In the practical setup this coupler will be placed in a polymer spacer base plate which is not shown in the basic schematic in Fig. 1. The excitation light is guided through the channel by means of reflections on the metallic-coated surfaces of the coupler and on the oblique sidewalls of the silicon channel. The excitation source and the detector for quantifying the amount of nonabsorbed excitation light can be placed off-chip and can be connected to the chip by optical fibers. Decoupling the optical part from the microfluidics eliminates the need of propagating a UV or VIS light beam in the silicon wafer plane. This way we open up the possibility of reusing the same optics for different microfluidic chips and of combining several couplers to probe a microfluidic channel simultaneously at different locations. Since the detection approach is independent of the microfluidic channel configuration we can build a generic detection system for parallel measurements on different types of microfluidic wafers. Finally also the fabrication of the optics and the 
microfluidics can be decoupled and the coupler can be mass produced in plastic by replication methods such as injection molding or hot embossing. Using a light coupler on top of a silicon microfluidic wafer for UV/VIS absorbance measurements is therefore a flexible and costeffective solution. We remark that this detection configuration is limited to silicon microfluidic wafers bonded to glass plates (as opposed to all-silicon devices) which contain microfluidic channels with oblique sidewalls at the required detection points.

The optical configuration requires a microfluidic channel with oblique reflecting sidewalls at the probing location. We have constructed a trapezoidal-shaped channel, using wet anisotropic etching in a silicon wafer [6]. The channel sidewalls have a slope of 54.74 degrees as determined by the anisotropic etching process in silicon. It has a channel width of $1.5 \mathrm{~mm}$ to provide a sufficiently large optical path length for absorbance detection. To limit the total detection volume the channel height is only $50 \mu \mathrm{m}$. The channel length over which the sample is analyzed equals $200 \mu \mathrm{m}$, the size of the light beam in the channel. These cross-section dimensions are chosen arbitrarily to construct this first proof-of-concept prototype, but can be changed to suit other applications. Four alignment marks are patterned around the channel. These will be used for aligning the micro-optics with respect to the chip. Holes with a diameter of $500 \mu \mathrm{m}$ were drilled through the bottom of the silicon chip, prior to the anodic bonding of a standard Pyrex 7740 glass plate with a thickness of $700 \mu \mathrm{m}$ to the silicon chip. The holes serve as an inlet and outlet on which Nanoports (Upchurch Scientific) are glued to provide a leakage-free connection between the channel, the external fluidic pumping system and the waste reservoir. As a first step, we used nonsequential optical ray tracing simulations (ASAP, Breault Research Organization, Inc) to model the system and to optimize its performance with respect to optical efficiency and system complexity in terms of the number and the type of optical components used. For the fabrication of micro-optical components we use Deep Proton Writing (DPW), the technology to rapidly prototype micro-optical systems that can combine micromechanical positioning structures and refractive micro-optical components [7].

Next, the fabricated and assembled microsystem is tested in a proof-of-concept demonstration setup (see Fig. 2 ). Excitation light generated by a violet laser diode (iPulse, $\lambda=405 \mathrm{~nm}, 10 \mathrm{~mW}$, Toptica) is on/off modulated at $521 \mathrm{~Hz}$ and guided to the microsystem by a SMF (SMF28, Corning). The nonabsorbed excitation light which has propagated through the microfluidic channel is coupled in a large core MMF (BFH48-600, Thorlabs). The light guided through the fiber is spectrally filtered with a band pass filter (D405/10, AHF Analysentechnik) to select only the excitation wavelength and to suppress possible fluorescence and environmental stray light. The filtered light is quantified by a silicon photodiode (818-UV, Newport). The analog photodiode voltage signal is filtered by two notch filters which remove noise at the line frequency $(50 \mathrm{~Hz})$ and twice the line frequency to clean up the detector signal.

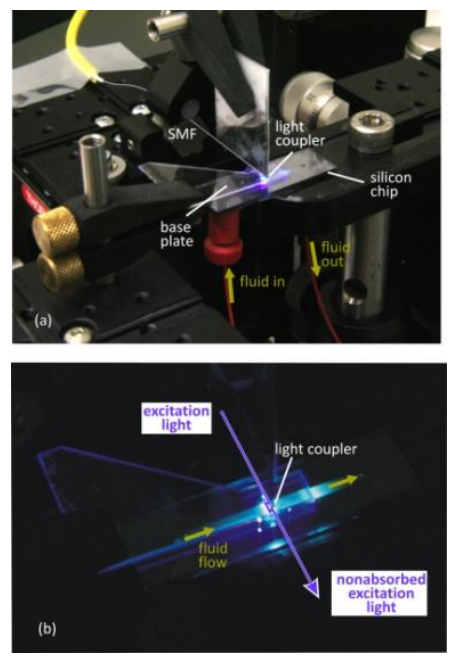

Fig. 2. Close-up of the microfluidic system in the proof-of-concept demonstration setup.

Afterwards the signal is fed to a lock-in amplifier (SR830, Stanford Research Systems) which extracts and quantifies the signal modulated at the same frequency as the excitation source. In this setup we observe a large noise component in the detector output around $1 \mathrm{kHz}$, which is not present at lower frequencies. Therefore the source is modulated at a lower frequency, here $521 \mathrm{~Hz}$. The settings of the noise filter in the lock-in amplifier can be adjusted to optimize the filtering of noise at frequencies very close to the reference frequency. Large filter time constants, corresponding to small filter bandwidths, provide efficient filtering. However they cause slow detector response and output smoothing. For this setup a trade-off was found experimentally for a filter with a time constant of $10 \mathrm{~ms}$ and $18 \mathrm{~dB}$ roll-off. The lockin amplifier voltage output is sampled at $10 \mathrm{~Hz}$, which is the highest sample rate possible in the current hardware configuration. In a practical application, the sample rate should be chosen in function of the smallest sample peaks that need to be analyzed so, that 25 data points per peak can be measured [8]. In this proof-of-concept demonstration we work with the highest sample rate available to enable measurements of various kinds of peaks. The amplified signal is read out through a GPIB connection by a computer using LabVIEW software and is further processed by means of Matlab.

The chemical samples under test are delivered to the 
microfluidic channel by a syringe pump (KD Scientific) connected to a high-pressure switching valve (Rheodyne) with a sample loop of 5 microliter. PEEK tubing (Upchurch Scientific) is used to connect the valve to one of the Nanoports glued on the silicon microfluidic chip. The other Nanoport is connected to a waste reservoir. To determine experimentally the concentration measurement range and the detection limit, the response of the system on a set of standard samples with a known concentration is measured. All samples are prepared from a stock solution consisting of 12 millimolar $(\mathrm{mM})$ coumarin 102 dye (Acros Organics) dissolved in methanol (Sigma Aldrich). Samples at lower concentrations are prepared by dilution of the stock solution with methanol.

Our goal is to use this detection system for quantitative analysis of the concentration of analytical samples. As we do not measure the concentration directly, we set up a calibration curve, which provides a mathematical relation between the output of the system (peak area of the absorbance signal [9]) and the sample concentration. As a case study in this letter we set up a calibration curve for the dye coumarin 102. Five standard solutions with concentrations between $0.6 \mu \mathrm{M}$ and $6 \mathrm{mM}$ are characterized (see example in Fig. 3).

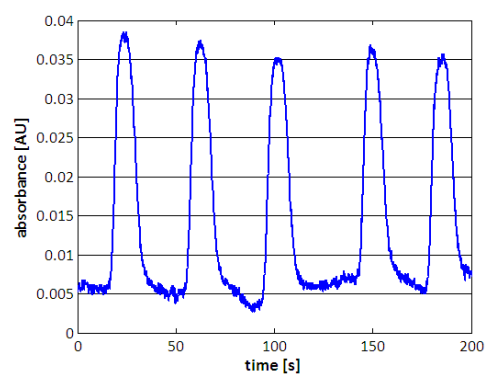

Fig. 3. Absorbance measurement of 5 consecutive injections of a coumarin 102 sample with a concentration of $60 \mu \mathrm{M}$.

\begin{tabular}{c|cccc}
\hline $\begin{array}{c}\text { Concentration } \\
(\mathrm{M})\end{array}$ & $\begin{array}{c}\text { Peak area } \\
{[\mathrm{Au} * \mathrm{~s}]}\end{array}$ & $\begin{array}{c}\text { SD } \\
{[\mathrm{Au} * \mathrm{~s}]}\end{array}$ & \% RSD & SNR \\
\hline $6 * 10^{-3}$ & $1.9283 * 10^{-1}$ & $3.7 * 10^{-3}$ & 2.0 & 20321 \\
$6 * 10^{-4}$ & $2.4513 * 10^{-2}$ & $7.4 * 10^{-4}$ & 3.0 & 3099 \\
$6 * 10^{-5}$ & $2.44 * 10^{-3}$ & $1.2 * 10^{-4}$ & 5.1 & 268 \\
$6 * 10^{-6}$ & $2.89 * 10^{-4}$ & $2.2 * 10^{-5}$ & 7.5 & 61 \\
$6 * 10^{-7}$ & $6.2 * 10^{-5}$ & $2.1 * 10^{-5}$ & 34 & 14 \\
\hline
\end{tabular}

Table 1. Average peak area measurements (in $\mathrm{AU}^{*} \mathrm{~s}$ ) with standard deviation (SD), relative standard deviation (\% RSD) and average signalto-noise ratio (SNR) (for each concentration average of 6 measurements)

For every concentration six consecutive injections are measured. After a time-domain filtering step (moving average filter), the linear background drift caused by the source, detector and possible solvent variations is fitted with a 1 st order curve and removed from the measured signal. This procedure is called trend-removal. Finally the signal is differentiated for peak identification. At the peak locations the filtered non-differentiated detector signal is further processed to quantify the areas of the measured sample peaks resulting in small standard deviations as shown in Table 1. We observe that the precision, represented by the standard deviation (SD), obtained for the replicated measurements is not constant for all concentrations, which is commonly observed when large concentration ranges are considered [10]. As a last step we applied weighted least squares regression techniques on the data points to obtain the mathematical representation of the calibration curve resulting in a detection limit of $4.3 \mu \mathrm{M}$. This is comparable to the performance reached with state-of-the-art miniaturized waveguide based detection systems. The limit of detection could even be further improved by reducing the amount of stray light in the system.

We can conclude that we have demonstrated the proofof-concept of this microfluidic light probe by measuring standard samples of coumarin 102 dye with concentrations between $0.6 \mu \mathrm{M}$ and $6 \mathrm{mM}$. Decoupling the optics from the microfluidics opens opportunities in terms of low-cost mass production of the optics and the development of generic detection systems for parallel measurements on different types of microfluidic circuits.

This work was supported in part by the DWTC-IAP, in part, by the FWO, in part by the GOA, in part, by the 6th FP European Network of Excellence on Micro-Optics (NEMO) and the 7th FP European Network of Excellence on Biophotonics (Photonics4Life), in part, by the Methusalem and Hercules foundations and, in part, by the OZR of the Vrije Universiteit Brussel.

\section{References}

[1] G.M. Whitesides, Nature 442, 368 (2006).

[2] A. Chandrasekaran, M. Packirisamy, Proc. of SPIE 7099, 70992B-1 (2008)

[3] E. Verpoorte, A. Manz, H. Lüdi, A.E. Bruno, F. Maystre, B. Krattiger, H.M. Widmer, B.H. van der Schoot, N.F. de Rooij, Sens. Act. B 6, 66 (1992).

[4] R.M. Tiggelaar, T.T. Veenstra, R.G.P. Sanders, J.G.E. Gardeniers, M.C. Elwenspoek, A. van den Berg, Talanta 56, 331 (2002).

[5] H. Salimi-Moosavi, Y. Jiang, L. Lester, G. McKinnon, D.J. Harrison, Electrophoresis 21, 1291 (2000).

[6] B. Ziaie, A. Baldi, M. Atashbar, Introduction to Micro/ Nanofabrication, in: B. Bhushan (Ed.), Handbook of Nanotechnology (B. Bhushan (Ed.), Springer, Berlin, 2007).

[7] C. Debaes, J. Van Erps, M. Vervaeke, B. Volckaerts, H. Ottevaere, V. Gomez, P. Vynck, L. Desmet, R. Krajewski, Y. Ishii, A. Hermanne, H. Thienpont, New J. Phys. 8, 270 (2006).

[8] C. Perrin, H. Fabre, D.L. Massart, Y. Vander Heyden, Electrophoresis 24, 2469 (2004).

[9] A. Felinger, Data analysis and signal processing in chromatography (Amsterdam, The Netherlands, Elsevier, 1998).

[10] Y. Vander Heyden, R. Boqué Rodríguez, M. Cuesta, Calibration, LCGC Europe 20 (2007). 